\title{
Article
}

\section{Increased distractibility in schizotypy: Independent of individual differences in working memory capacity?}

Marsh, John Everett, Vachon, F and Sörqvist, P

Available at http://clok.uclan.ac.uk/19706/

Marsh, John Everett ORCID: 0000-0002-9494-1287, Vachon, $F$ and Sörqvist, P (2017) Increased distractibility in schizotypy: Independent of individual differences in working memory capacity? Quarterly Journal of Experimental Psychology, 70 (3). pp. 565-578. ISSN 1747-0218

It is advisable to refer to the publisher's version if you intend to cite from the work. http://dx.doi.org/10.1080/17470218.2016.1172094

For more information about UCLan's research in this area go to http://www.uclan.ac.uk/researchgroups/ and search for <name of research Group>.

For information about Research generally at UCLan please go to http://www.uclan.ac.uk/research/

All outputs in CLoK are protected by Intellectual Property Rights law, including Copyright law. Copyright, IPR and Moral Rights for the works on this site are retained by the individual authors and/or other copyright owners. Terms and conditions for use of this material are defined in the policies page.

\section{CLoK}

Central Lancashire online Knowledge www.clok.uclan.ac.uk

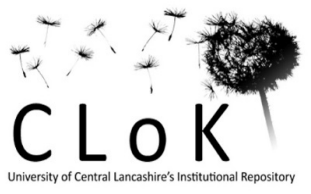


Increased Distractibility in Schizotypy:

Independent of Individual Differences in Working Memory Capacity?

John E. Marsh ${ }^{1,2}$, François Vachon ${ }^{3} \&$ Patrik Sörqvist $^{2}$

${ }^{1}$ School of Psychology, University of Central Lancashire, Preston, UK.

${ }^{2}$ Department of Building, Energy and Environmental Engineering, University of Gävle,

Gävle, Sweden

${ }^{3}$ École de psychologie, Université Laval, Québec, Canada

RUNNING HEAD: Increased Distractibility in Schizotypy

Correspondence: John E. Marsh, School of Psychology, Darwin Building, University of Central Lancashire, Preston, Lancashire, United Kingdom, PR1 2HE.

Phone (+44) 1772 893754, Fax (+44) 1772892925

E-mail: JEMarsh@uclan.ac.uk 
Increased Distractibility in Schizotypy: Independent of Individual Differences in Working Memory Capacity?

\begin{abstract}
Individuals with schizophrenia typically show increased levels of distractibility. This has been attributed to impaired working memory capacity (WMC), since lower WMC is typically associated with higher distractibility and schizophrenia is typically associated with impoverished WMC. Here, participants performed verbal and spatial serial recall tasks that were accompanied by to-be-ignored speech tokens. For the few trials wherein one speech token was replaced with a different token, impairment was produced to task scores (a deviation effect). Participants subsequently completed a schizotypy questionnaire and a WMC measure. Higher schizotypy scores were associated with lower WMC (as measured with operation span [OSPAN]), but WMC and schizotypy scores explained unique variance in relation to the mean magnitude of the deviation effect. These results suggest that schizotypy is associated with heightened domain-general distractibility, but that this is independent of its relationship with WMC.
\end{abstract}

Keywords: Schizophrenia, Schizotypy, Distraction, Domain-Specificity, Verbal Working Memory, Visuo-Spatial Working Memory, Working Memory Capacity 


\section{Introduction}

Selective attention is necessarily a fine balance between being receptive to environmental change - if that information becomes more important than the current focal task activity — while avoiding being so maladaptively focused that no environmental change whatsoever triggers attentional capture. However, certain attentional disorders suggest a suboptimal attentional selectivity, such as attentional deficit hyperactivity disorder (ADHD; Pelletier, Hodgetts, Lafleur, Vincent, \& Tremblay, 2014). Many researchers recognize that one of the core features of schizophrenia is reduced attentional control or so-called attentional deficit (Andreasen, 1999). The concomitant, fundamental dysfunction of selective attention among individuals with the disorder is reported by both clinicians and patients (MacDonald, 1960; McGhie \& Chapman, 1961) and there is suggestion that such attentional dysfunction may contribute to other symptoms of the disease such as impaired social functioning (Mirsky \& Duncan, 1986). Moreover, another core feature of the disorder is dysfunction in working memory processing (for a review, see Lee \& Park, 2005). Deficits have been found in the context of verbal short-term memory tasks (e.g., Bell, Byson, \& Wexler, 2001; Oltmanns \& Neale, 1975) and visuo-spatial short-term memory tasks (e.g., Cellard, Tremblay, Lehoux, \& Roy, 2007; Smith, Park, \& Cornblatt, 2006; for an overview, see Park \& Gooding, 2014). However, very few attempts have been made to combine selective attention deficits and working memory research, and to our knowledge none of these has investigated cross-modal distraction in relation to schizophrenia or schizotypy.

In this paper, we explore the propensity for a rare auditory event to capture attention — as indexed by impoverished performance on visually-based serial recall tasks_-for individuals that possess schizophrenic traits. To achieve this goal, we adopt a continuum approach to psychosis by measuring schizotypy or psychosis-proneness scores (e.g., Claridge, 1997). A well-supported concept within the literature is that parallels in behaviours, 
personality characteristics (e.g., psychotic tendencies), and belief structures, evident in potentially hospitalized patients with schizophrenia, are also displayed by healthy individuals within the normal population (albeit fewer and less pronounced). Indeed, the dimensions of schizotypy and schizophrenia are very similar (for a review, see Vollema \& van den Bosch, 1995). More crucially, however, is that the processing dysfunctions in schizotypy parallel those found in schizophrenia with regard to cognitive (Poreh et al., 1995), neuro-cognitive (Rawlings \& Goldberg, 2001), and psychophysiological (O'Driscoll et al., 1998) domains. These dysfunctions in individuals that have schizotypal characteristics, and relatives of patients with schizophrenia whom are at high risk of developing the disorder, include deficits in relation to attention (Braff, 1993). Similarly, twin studies of schizophrenic probands reveal that working memory deficits increase as the degree of genetic overlap between the affected proband increases (Pirkola et al., 2005). While some studies have shown that the deficits appear to be independent of medication (Carter et al., 1996), others have demonstrated both improvements and impairments in working memory performance with different antipsychotic medications (McGurk et al., 2005).

Several advantages are associated with studying high-schizotypy but healthy volunteers as compared with patients. In addition to being more accessible for experimental research, healthy participants can self-report their feelings and experiences with greater accuracy than can schizophrenic patients. Moreover, examining schizotypy in healthy individuals circumvents confounding factors such as long-term medication (which may reduce the extent to which processing dysfunctions manifest), overt symptoms, hospitalization, and diagnostic stigma (Mednick \& McNeil, 1968; O'Flynn et al., 2003), thereby rendering it potentially possible to identify trait markers of attentional/processing dysfunction in patients with schizophrenia. Here, we investigate cross-modal distraction as a vehicle to understand the attentional processing dysfunctions in schizotypy. 


\section{Within-Modality Distraction}

Before we turn to cross-modal distraction, we first give a brief history of withinmodality distraction effects and point to the advantage of using cross-modal distraction instead. Within-modality distraction effects have been extensively assessed in the schizotypy and schizophrenia literature, but the results are difficult to interpret. For example, individuals scoring high on schizotypy do not typically show increased Stroop interference (Kaplan \& Lubow, 2011). This may be because classic selective attention tasks such as Stroop comprise task-relevant stimuli (the colour name) and task-irrelevant stimuli (the overlearned colour word) in a way in which they are spatially integrated within the same object. Within such a setting, strong interference may render the task impervious to modulation via factors such as schizotypy (Kaplan \& Lubow, 2011). Therefore, differences between high and low schizotypy could simply be attributable to differences in attention toward the target rather than to differences in the ability to control distracter interference.

Moreover, in tasks within which sequences of to-be-remembered auditory items are interleaved with auditory distractor items (Oltmanns, 1978) or sequences of visual-spatial locations are interleaved with a distracter location (Cellard et al., 2007), impairment may also be attributable to within-modality shifts of attention. Therefore, a paucity of focused attention to target relevant locations within the visual environment, perhaps due to difficulty in distinguishing relevant from irrelevant stimuli (Gray, Feldon, Rawlins, Hemsley, \& Smith, 1991), could determine the processing impairments associated with schizotypy. When relevant and irrelevant stimuli are presented in the same, visual, modality it is sometimes the case that distractors can be excluded from the visual field. Moreover, when relevant and irrelevant stimuli are presented in the auditory modality, some sounds can impair the processing of others at a peripheral level due to sensory confusion or energetic masking. Within these contexts, shifting attention to irrelevant information within a modality could 
arguably cause performance disruption. One way in which the potential shortcomings of within-modality distraction can be minimised is to instead use cross-modal distraction to assess differences in attentional selectivity in schizophrenia or high-schizotypy individuals.

\section{Cross-Modal Distraction: The Deviation Effect in Short-Term Memory}

In contrast to vision where one can curtail the processing of unwanted or irrelevant stimuli by shutting one's eyelids or averting one's case, there is no simply physical means by which the same feat can be achieved with audition. Unlike visual information, whereby the eyes require directing toward the source of the stimulus, sound is always within the individual's sensory "reach": Even if people's visual attention is focused elsewhere, they are preattentively processing the auditory environment surrounding them, in an obligatory fashion that is automatic and beyond the control of the individual. Therefore, distraction produced by auditory events when an individual's attention is directed to a visual task cannot (easily) been explained by a failure to maintain attention (e.g., on a spatial location). The obligatory processing of regularities in the auditory environment yields the capacity for attentional capture by the onset of a rare sound that deviates from this pattern.

The deviation effect refers to the performance decrement that is produced by an infrequent and typically unexpected change (deviation) in an otherwise repetitive or continuous auditory input. Deviation produces marked disruption of visual-verbal serial recall and other, usually visual-verbal, short-term memory tasks (Hughes, Vachon, \& Jones, 2005, 2007). For example, a sound sequence with a single deviant (e.g., a a a a b a a a), that is presented at the same time as a sequence of to-be-recalled items, typically impairs recall to a greater degree than a sound sequence that does not contain a deviant (e.g., a a a a a a a). A deviation within an otherwise expected pattern of auditory stimulation is thought to produce an orienting response (OR; Cowan, 1995; Sokolov, 1963; Voronin \& Sokolov, 1960). Theoretically, the OR occurs only when an incoming stimulus mismatches a mental 
representation - a neural model (cf. Sokolov, 1963) — of that stimulus which is progressively fashioned as the stimulus sequence unfolds (Cowan, 1995; Schröger, 1997; Vachon, Hughes, \& Jones, 2012). The OR is associated with the attentional focus being captured involuntarily away from the visual-verbal focal task toward the deviant event, the consequence of which is reduced focal task performance (Näätänen, Tervaniemi, Sussman, Paavilainen, \& Winkler, 2001). As such, the deviation effect is a tool suitable for studying how schizotypy underpins distractibility without the potential confound of sensory confusion or energetic masking.

\section{Individual Differences in Susceptibility to Distraction Underpinned by ORs}

Many attentional dysfunctions in schizophrenia have been linked to anomalies of attentional orienting and habituation (Hemsley, 1987). For example, there is evidence that habituation to (or suppression or gating of) the startle reflex, when a weak non-startling stimulus follows an intense stimulus, is abnormal (reduced) in schizophrenia and schizotypy (Cadenhead, Geyer, \& Braff, 1993; Croft, Lee, Bertolot, \& Gruzelier, 2001; Evans, Gray, \&

Snowden, 2007). Moreover, positive symptoms — such as unreality or unusual experiences-a subtype of the syndrome represented by schizophrenia (O'Flynn et al., 2003), may be associated with particular features of the disorder that relate to increased novelty detection (and therefore more pronounced ORs) and deficient habituation to ORs. That is, impoverished habituation may bear syndromal relations (Vernon et al., 2005). Finally, another subtype of symptoms, impulsivity, is associated with the degree to which attention is fixed on a current source of input. Compared to low-impulsive individuals, high-impulsive individuals can shift their attention more easily from one location to another (Dickman, 1993, 2000). Impulsivity, although advantageous in many cognitive tasks that require shifting of attention with very little forethought (Dickman, 1993, 1996) may be disadvantageous in the context of cross-modal distraction since a relative impulsivity to attend to a change in the 
auditory scene could lead to a pronounced OR and, as a consequence, prolongation of the orienting required to re-engage with the visually-based focal task.

Another individual differences variable - aside from schizotypy — that has been associated with attentional control (and with cross-modal auditory distraction in particular) is working memory capacity (WMC). For example, high-WMC individuals are more able than their low-WMC counterparts to inhibit undesired eye saccades (Kane, Bleckley, Conway, \& Engle, 2001) and reflexive responses in the context of the Stroop task (Kane \& Engle, 2003). Based on findings such as these, the executive attention view of WMC has emerged (Engle, 2002), suggesting that the key difference between high- and low-WMC individuals is their ability to control attention and stay focused on the task at hand in the face of distraction. Individual differences in attention control capabilities — as indicated by $\mathrm{WMC}$ - are related to individual differences in susceptibility to the deviation effect (Sörqvist, Marsh, \& Nöstl, 2013). High WMC-individuals are not as impaired by an auditory deviant as their low-WMC counterparts (Hughes, Hurlstone, Marsh, Vachon, \& Jones, 2013; Sörqvist, 2010). Moreover, habituation of the $\mathrm{OR}$ - as indexed by the effects of unexpected sound on task performanceis related to individual differences in WMC, with low-WMC individuals showing impoverished habituation (Sörqvist, Nöstl, \& Halin, 2012). Generally, low-WMC individuals are more susceptible to cross-modal distraction because they show impoverished auditorysensory gating of the task irrelevant background sound (Sörqvist, Stenfelt, \& Rönnberg, 2012) and because they fail to withhold the locus-of-attention upon the target modality when an auditory deviant is presented (Sörqvist et al., 2013; Sörqvist \& Marsh, 2015).

\section{Present study}

The higher susceptibility to distraction found in both high-schizotypy and in lowWMC individuals is consistent with the finding that WMC is typically impoverished in highschizotypy individuals (Farmer et al., 2000; Siever et al., 2002). In this respect, one 
possibility is that, like with many abnormalities that co-vary with inferior WMC (Sörqvist \& Rönnberg, 2014), the low WMC of high-schizotypy individuals underpins their greater susceptibility to distraction. This hypothesis is tested in the experiment reported here in two ways. First, by using a median split of participants based upon their overall schizotypy scores - as measured by the Oxford-Liverpool Inventory of Feelings and Experiences (OLIFE) — and investigating whether any potential differences in distraction were present in the absence of any difference in WMC. Second, by obtaining individual difference scores in WMC and in schizotypy and have these two variables compete-in the context of a regression model — for the explanation of variance in the magnitude of the deviation effect. We also made a detailed analysis of the explanatory power of four subscales of schizotypy (Cognitive Disorganization, Introvertive Anhedonia, Impulsive Nonconformity, and Unusual Experiences) as they have previously been associated with functionally different attentional deficits as detailed above.

Moreover, the domain-generality of the distractibility in high-schizotypy individuals was also explored by testing the effects of presenting an auditory deviant on visuo-spatial serial recall and visual-verbal serial recall. Spatial working memory and verbal working memory are neurologically dissociable (Smith, Jonides, \& Koeppe, 1996) and the working memory deficit found in schizophrenics appears to be modality independent (although there is some evidence suggesting that spatial working memory is more greatly impaired than verbal; see Lee \& Park, 2005). Here, we wish to capture the global (i.e. amodal) susceptibility to distraction by task-irrelevant stimulation with as little influence as possible from task domains or requirements. To do so, we extracted an index of the size of the distraction effect compounded across the verbal and spatial domains. 


\section{Method}

\section{Participants}

Ninety non-smoking native English speakers (66 female, 18 to 35 years of age, mean age $=22.3, S D=4.8$ ) reporting normal hearing and normal or corrected-to-normal vision were recruited from the University of Central Lancashire. None of the participants reported any neurological or psychiatric disorders. Participants received a small honorarium for taking part. Due to experimenter error (2), equipment failure (3) and an obvious miscomprehension of written task instruction (1), six participants were removed, thereby yielding a sample size of 84 (61 female, 18-35 years of age, mean age $=22.2, S D=4.8)$.

\section{Materials}

To-be-ignored auditory sequences. For the irrelevant auditory sequences, two sets of 10 spoken letters ( $A B C G J K L M Q S$ ) were recorded in a female voice. Within each set, the letters were recorded in an even pitch and recorded to 16-bit resolution at $22 \mathrm{kHz}$ sampling rate using Sony Sound Forge 8.0 software. They were each edited to a duration of $250 \mathrm{~ms}$.

Two types of to-be-ignored auditory sequence were created: In 'no-deviant' sequences, a sequence of 10 same letter tokens (i.e., 'k k k k k k k k k k') was presented). For deviant trials, a different, randomly chosen letter (e.g., 'm') from the set was presented in the fourth position of the 10-letter sequence (e.g., ' $\mathrm{k} \mathrm{k} \mathrm{k} \mathrm{m} \mathrm{k} \mathrm{k} \mathrm{k} \mathrm{k} \mathrm{k} \mathrm{k').} \mathrm{Thus,} \mathrm{the} \mathrm{deviant} \mathrm{sound}$ was a verbal deviant, corresponding to a change in letter identity. The auditory sequences were presented via headphones at a sound level of approximately $65 \mathrm{~dB}(\mathrm{~A})$.

Visuo-verbal serial recall task. The visual to-be-remembered lists comprised seven digits drawn from the set 1-7. These digits were sampled without replacement and were arranged in a quasi-random order with the constraint that there were no ascending or descending runs of more than two digits. These digits were presented in black Times New 
Roman font on a white background, one at a time, sequentially, in the central position of a computer display. Each item was $2.39^{\circ}$ in height. The digits were presented for $450 \mathrm{~ms}$ each, with a 400 ms inter-stimulus interval (see Figure 1). There were 45 trials in total: 39 'no deviant' trials and 6 'deviant' trials. A deviant occurred on Trials 5, 8, 18, 27, 35, and 41 within each block. The onset of the to-be-ignored auditory sequence preceded the onset of the first to-be-remembered item by $125 \mathrm{~ms}$, with a $300 \mathrm{~ms}$ inter-stimulus interval between each spoken letter. Thus, the irrelevant sequence was presented synchronously with the visual sequence presentation. Using these timing parameters, the verbal-deviant on 'deviant' trials occurred $125 \mathrm{~ms}$ before the onset of the third to-be-remembered item.

Figure 1 about here

The task began with two practice trials performed in quiet prior to the block of 45 experimental trials. Participants initiated a trial by pressing the spacebar. For the visualverbal serial recall task, $400 \mathrm{~ms}$ after the presentation of the last item in each trial the mouse pointer appeared in the centre of the screen with all of the to-be-remembered digits presented horizontally and arranged canonically. Participants were required to reconstruct the order by using a mouse-driven pointer to click on the digits in the order they were presented. Once selected, a digit changed from black to green. No omissions or repetitions were permitted. Once the participant had recalled the whole sequence, they pressed the spacebar to begin the next trial. No sound was presented during the response stage of the tasks.

Visuo-spatial serial recall task: dot task. In each trial, a sequence of seven black dots measuring approximately $0.81^{\circ}$ in diameter was presented at different spatial locations within a white screen measuring $16.5 \times 16.5^{\circ}$. The coordinates for the dots was randomly generated across seven spatial locations. The centers of any pair of dots were separated by a distance of between $2.86^{\circ}$ and $10.07^{\circ}$. No dot appeared closer than $2.86^{\circ}$ to the centre point of the 
presentation screen. The presentation duration for each dot was on for $450 \mathrm{~ms}$ and off for 400 ms (see Figure 1). The number of trials, occurrences of deviants, the synchronicity between auditory and visual stimuli, and so on, was identical for the verbal and the visuo-spatial serial recall tasks.

For the visuo-spatial serial recall task, $400 \mathrm{~ms}$ after presentation of the last dot in each trial, all of the dots reappeared in their original spatial locations. Participants were required to use a mouse-driven pointer to click on the dots in the order they were presented. The colour of a selected dot changed from black to green after it had been selected. No omissions or repetitions were allowed. Once the participant had recalled the whole sequence, they pressed the spacebar to begin the next trial. No sound was presented during the response stage of the tasks.

WMC measure. The automatic operation span (AOSPAN) task was used to assess WMC (Unsworth, Heitz, Schrock, \& Engle, 2005). AOSPAN is a standardized measure of WMC and involves encoding and recalling a letter sequence while simultaneously solving mathematical operations (for details, see Unsworth et al., 2005). For example, on this task, participants are presented with visual lists of letters (sampled at random from the set: F, H, J, $\mathrm{K}, \mathrm{L}, \mathrm{N}, \mathrm{P}, \mathrm{Q}, \mathrm{R}, \mathrm{S}, \mathrm{T}$, and $\mathrm{Y}$ ) that they are later required to recall in serial order. Each letter presentation is followed by a mathematical operation [e.g., $(8+3) \times 2$ ?] that participants are required to solve as quickly and as accurately as possible. Immediately after participants solved the operation, they clicked the left mouse button and a solution was displayed onscreen. Participants were then required to indicate whether the given solution to the operation was correct or not, by clicking the mouse on a "True" or "False" icon. After responding, the next letter was presented, followed by another mathematical operation, and so on. Once all the list-items had been presented, the letters were displayed at random positions in a $4 \times 3$ spatial array, and participants were required to click on the letters presented in forward serial 
order using the mouse. The list-length and hence the number of intervening mathematical operations varied from three to seven. There were three trials for each list-length, and lists were administered in a different random order for each participant. Before commencing the task proper, participants received a set of practice trials in which they engaged in the serial recall task only, followed by practice trials in which they solved mathematical operations only, before finally engaging in practice trials in which the serial recall and mathematical operations were combined.

Schizotypy measure. The self-report, dichotomous choice scale, O-LIFE (Mason, Claridge, \& Jackson, 1995) was used to measure four dimensions of schizotypy: cognitive disorganization (attentional difficulties, social anxiety and emotional sensitivity), impulsive nonconformity (reckless and destructive tendencies), introvertive anhedonia (so-called negative schizotypy reflecting inability to experience pleasure and lack of social contact), and unusual experiences (so-called positive schizotypy representing deviant perceptual and cognitive experience). Twenty-five questions each tapped four different subscales.

\section{Design and Procedure}

An experimental within-participants design with trial type (deviant present vs. deviant absent) as the independent variable was used to obtain individual difference scores for susceptibility to distraction. These individual difference scores were analysed by correlational means by using individual difference scores in WMC and O-LIFE as predictors.

The experiment was executed on a PC running an E-Prime 2.0 program that controlled stimulus presentation. The participants began with the two serial recall tasks. They were conducted in separated blocks (one block for the verbal task, one block for the spatial task). The two tasks were administered with a brief, optional pause between, and their order was counterbalanced across participants: half participants started with the visual-verbal task and half started with the visuo-spatial task. At the beginning of each block, participants read 
standard instructions which told them to recall the order of presentation of the seven digits in the "verbal" block and the presentation order of the seven dots in the "spatial" block. They were also informed that sounds would be presented over their headphones but that they were irrelevant to the recall task and that they should ignore it as best as they could. They were not told about the presence of deviant events within the sound. After the two serial recall tasks, the participants conducted the AOSPAN (henceforth "OSPAN") task and answered the OLIFE questionnaire.

\section{Results}

Regarding serial recall performance, the raw data was scored according to the strict serial recall criterion: Responses were only recorded as correct if the item, or dot, appeared in its original position of presentation. Below, the serial recall scores are expressed as percentages of the maximum. Performance was compared across two groups created using a median split of overall schizotypy scores. Means for the six individual difference variables are found for each group in Table 1 and intercorrelations between them are found in Table 2 .

\section{The deviation effect in cross-modal serial recall}

As can be seen in Figure 2, the auditory deviant captured attention-as indexed by a reduction in serial recall performance - and this effect was larger for high schizotypy participants in comparison with low schizotypy participants. These general conclusions were confirmed by a 2 (Schizotypy group: high vs. low) $\times 2$ (Trial type: no deviant vs. with deviant $) \times 2$ (Type of task: spatial serial recall vs. verbal serial recall) mixed ANOVA. Of note, the same conclusions arise when OSPAN scores are added to the analysis as a covariate. Because of this, only results without OSPAN as a co-variate are reported below. The analysis revealed a significant main effect of Schizotypy group, $F(1,82)=6.29, p=.014, \eta_{\mathrm{p}}^{2}$ $=.07$, a significant main effect of Trial type, $F(1,82)=13.53, p<.001, \eta_{\mathrm{p}}{ }^{2}=.14$, and a significant main effect of Type of task, $F(1,82)=30.07, p<.001, \eta_{\mathrm{p}}^{2}=.27$. There was also a 
significant interaction between Trial type and Schizotypy group, $F(1,82)=12.16, p=.001$, $\eta_{\mathrm{p}}^{2}=.13$, suggesting that the deviation effect was larger for high schizotypy participants. There was no significant interaction between Type of task and Schizotypy group, $F(1,82)=$ $0.85, p=.358, \eta_{\mathrm{p}}{ }^{2}=.01$, and no significant three-way interaction between all three variables, $F(1,82)=0.41, p=.524, \eta_{\mathrm{p}}^{2}=.005$.

The analysis revealed a significant interaction between Trial type and Type of task, $F(1,82)=10.79, p=.002, \eta_{\mathrm{p}}^{2}=.12$, which showed that the deviation effect was larger in the context of the verbal serial recall task. One possibility as to why the deviation effect was less pronounced in the visuo-spatial task is that it entails greater task difficulty, a factor known to reduce the deviation effect in visual-verbal serial recall (Hughes et al., 2013). Indeed, further analysis demonstrated that the superiority of verbal serial recall over visuo-spatial serial recall became less pronounced with higher WMC (as given by the correlation between OSPAN scores and the difference scores obtained when performance in visuo-spatial serial recall is subtracted from verbal serial recall in the no-deviant conditions, $r[82]=-.28, p=$ .011). This suggests that efficient visuo-spatial serial recall requires greater WMC, or executive resources, than efficient verbal serial recall does (Miyake et al., 2001).

Figure 2 about here

\section{Individual difference variables as predictors of the deviation effect}

A mean magnitude of the deviation effect across both tasks was calculated by averaging the difference scores between the deviant trials and the no-deviant trials in the two tasks respectively. The mean was $0.047(S E=0.01$, range $-0.24-0.38)$ which differed significantly from 0 (i.e., a statistically confirmed deviation effect), $t(83)=3.45, p<.001$. A multiple regression analysis was conducted to test whether the increased susceptibility to distraction in high schizotypy individuals depends on co-variation with WMC, or rather 
whether WMC and schizotypy each explain unique/independent portions of the variance. Multiple regression analyses control for co-variance between independent variables and, hence, enable the researcher to conclude that any explained variance by any of the independent variables is unique variance.

For the regression analysis, the mean magnitude of the deviation effect was selected as the dependent variable, and OSPAN scores and overall schizotypy was selected as the independent variables. The relationship between deviation effect size and both OSPAN and schizotypy scores is illustrated in Figure 3. The regression model was significant, $R^{2}=.26$, $F(2,81)=14.16, p<.001$. Higher OSPAN scores were associated with a smaller deviation effect, $\beta=-.30, p=.003$, and higher overall schizotypy was also associated with a greater deviation effect, $\beta=.35, p=.001$. WMC uniquely explained about $9 \%$ of the variance and schizotypy uniquely explained about $12 \%$ of the variance. The most important implication of this result is that increased distractibility in high schizotypy individuals does not depend on a co-variation with WMC.

Figure 3 about here

To further determine how schizotypy underpins distractibility, another multiple regression analysis was conducted with the mean magnitude of the deviation effect as the dependent variable and the four schizotypy subscales as the independent variables. Figure 4 shows the relationship between the size of the deviation effect and each of the four schizotypy subscale scores. The regression model was significant, $R^{2}=.29, F(4,79)=8.12, p$ $<.001$. Higher values on the unusual experiences scale was associated with higher distractibility, $\beta=.44, p<.001$, uniquely explaining about $19 \%$ of the variance. Higher values on the impulsive nonconformity subscale was also associated with higher distractibility, $\beta=.35, p=.004$, explaining another $12 \%$ of unique variance. Neither 
introvertive anhedonia, $\beta=-.02, p=.866$, nor cognitive disorganization, $\beta=-.16, p=.269$, was significantly related to the magnitude of the deviation effect. The same pattern was found when WMC was included within the regression.

Figure 4 about here

\section{Discussion}

A median split analysis showed that the magnitude of the deviation effect was larger for the high schizotypy participants in comparison with the low schizotypy participants. Higher schizotypy was associated with lower WMC - a factor known to be associated with increased susceptibility to the deviation effect (Hughes et al., 2013; Sörqvist, 2010; Sörqvist et al., 2012, 2013) — but this systematic difference in WMC between high and low schizotypy groups could not fully explain why the two groups differed in susceptibility to the deviation effect. A multiple regression analysis found that overall schizotypy explained a unique set of variance in susceptibility to the deviation effect, independent of the part of variance explained by individual differences in WMC. Moreover, the absence of a three-way interaction between task, schizotypy group and trial type suggests that the enhanced distractibility in high schizotypy is a domain-general rather than a domain-specific dysfunction.

Generally the findings are consistent with the idea that high schizotypy, like schizophrenia, is associated with an impairment in the ability to ignore irrelevant information (Fuentes, 2001; Fuentes, Boucart, Vivas, Alvarez, \& Zimmerman, 2000; Lipp \& Vaitl, 1992; Lubow, Ingberg-Sachs, Zahlstein-Orda, \& Gewirtz, 1992). The basis of this effect may lie within a deficit in sensory-motor gating and habituation (Braff, Geyer, \& Swerdlow, 2001) or a failure to inhibit the OR. This is consistent with the findings that attentional deficits in schizophrenia manifest when the task requires top-down involvement in task processing (cf. Gold et al., 2007; Nestor \& O’Donell, 1998; Van den Bosch, 1995). Top-down processing, or 
task-engagement, is known to reduce the distraction produced by a deviant (Hughes et al., 2013; Sörqvist \& Marsh, 2015). Moreover, that we have established increased distractibility in a cross-modal distraction task among high schizotypes rules out the alternative suggestion that the increased distractibility that has been previously demonstrated among this population (e.g., Cellard et al., 2007; Oltmanns, 1978) is associated with within-modality attentional shifts of attention.

\section{Impulsivity and Unusual Experiences (Positive Schizotypy)}

The contributions from separate subscales were also investigated. Higher values on the impulsive nonconformity subscale and on the unusual experiences subscale were systematically associated with a greater susceptibility to the deviation effect. In turn, introvertive anhedonia and cognitive disorganization were not related to distractibility. The relationship between schizotypy and the deviation effect is independent of any contribution played by WMC since schizotypy scores explained independent variance when assessing the susceptibility to distraction by the auditory deviation.

As impulsivity and unusual experiences explained a unique set of the variance in the magnitude of the deviation effect, the findings suggest that two functionally distinct mechanisms contribute to the enhanced distractibility in high schizotypes. Impulsivity, as tapped by the impulsive nonconformity scale, is associated with the extent to which attention is fixed on the present source of environmental input. Impulsivity is associated with the capability to shift attention more easily from one location, or stimulus, to another (Dickman, 2000). This ability is clearly of some advantage when a task requires shifting of attention without much by way of deliberate cognitive process. However, impulsive individuals may also experience tension within selective attention tasks whereby the balance between being receptive to changes in the environment and being steadfastly focused on the task at hand, is tipped toward being too receptive. In this way, subtle environmental changes may trigger 
attentional capture more for highly impulsive individuals resulting in a cost to performance as attention then requires re-orienting to the focal task. In this way, impulsivity would appear to have both functional — allowing the individual to switch attention easily between environmental inputs — and dysfunctional — requirement to reorient after an attentional switch-facets.

Unusual experiences, or positive schizotypy, associated with susceptibility to the deviant within the context of the serial recall task reported here, has been linked to deficiencies in fashioning a neural modal of an auditory stimulus (Vernon et al., 2005). According to Vernon et al. (2005), the neural model is maintained within short-term/working memory where a continuous matching process is occurring between the neural model and the incoming auditory stimulus (Siddle, 1991). Vernon et al. (2005) propose that this neural model becomes vulnerable to degradation through impoverished updating or rehearsal processes so that it is no longer a viable match for the incoming auditory stimulus. As a consequence, an OR will occur and habituation of the OR will diminish or fail (Öhman, Nordby, \& d'Elia, 1986). While our experiment did not examine habituation directly, it is important to address whether particular schizotypal symptoms are associated with a failure to suppress the OR, in addition to habituating the OR. One possibility is that the neural model for individuals scoring high on the unusual experiences scale of schizotypy lacks abstraction (or is impoverished) and is therefore associated with a failure to identify incoming stimuli resulting in a pronounced OR response when first encountering a deviant (and thereafter paucity of habituation of the OR). There has been some speculation that ideas of reference and other schizotypal symptoms such as unusual perceptual experiences (such as mistaking a sound for a voice or hallucinations) may be the manifestation of a failure to identify incoming stimuli (Raine et al., 1997; Vernon et al., 2005). The positive symptoms of schizophrenia, therefore, may be linked to the poor fashioning of a neural model. If this is indeed the case, 
then it is likely that studies that tap habituation processes more directly (e.g., Vachon et al., 2012) will be better able to empirically capture this possible deficiency.

However, evidence from processing auditory stimuli during visual attention in patients with schizophrenia suggests that the mismatch negativity (MMN) component of the event-related potentials (an index of the automatic detection of the deviant) differs from that of controls. Specifically, the MMN amplitude to pitch deviants (rare presentation of an 1122 $\mathrm{Hz}$ or a $1414 \mathrm{~Hz}$ tone embedded in a sequence of identical tones of $1000 \mathrm{~Hz}$ ) was smaller for schizophrenic patients, as compared with controls, while participants were engaged in a demanding visual discrimination task (Alain, Hargrave, \& Woods, 1998). This suggests that the representations of the standard stimulus from which the deviant differs is imprecise or more prone to decay for the schizophrenics (Alain et al., 1998). One implication of this is that due to an impoverished neural template of the sound pattern, schizophrenic patients should show a reduced deviation effect but also reduced habituation to the deviation effect. If high schizotypes in our study behaved similarly to schizophrenic patients, then we would have expected a reduced deviation effect and impoverished habituation to the deviant (as well any potential disruption to the standard repeated [steady-state] sequence). That is, if positive schizotypy was related to difficulties in fashioning a neural model of the auditory stimulation, then positive schizotypy could be associated with a reduced deviation effect (since a neural model is necessary for observing a deviant effect) and increased distraction from the repeated stimulus due to a failure to habituate to the standard. In support of this suggestion, Vernon et al. (2005) showed that high schizotypy individuals exhibited significantly less habituation suggesting that they can habituate but with more difficulties than low schizotypy individuals.

While high schizotypes performed more poorly in general than low schizotypes, without the inclusion of a quiet condition this cannot be taken as clear evidence for poorer habituation. Moreover, the finding that high schizotypes showed a more pronounced 
deviation effect suggests that they were able to fashion a neural model of the repeated stimulus. This appears to be at odds with the view that the neural model is somehow impoverished in individuals with high schizotypy. However, it is worth pointing out that substantial differences exist between our study and previous studies. For example, Alain et al. (1998) used tones as to-be-ignored sequences while letters were used in the current study. A letter change is more acoustically complex and salient than a change along a simple physical dimension such as a change in pitch. Moreover, Alain et al. (1998) demonstrated that a pattern deviant (e.g., an unexpected repetition of either a $500 \mathrm{~Hz}$ or $2000 \mathrm{~Hz}$ tone in an otherwise alternating sequence) was not associated with a diminished MMN, hence more salient deviants might elicit a similar MMN magnitude in both high and low schizotypes and patients with schizophrenia or their controls. One must also be cautious, however, about interpreting a diminished event-related brain potential as an index of reduced behavioural attentional capture because a behavioural-electrophysiological dissociation is sometimes reported. For example, Cortinas et al. (2008) showed that schizophrenic patients compared to controls showed an increased behavioural distraction, but a reduced novelty P3, to a deviant complex novel tone among to-be-ignored repetitive standard tones when engaged in a visually-based categorisation task. The novelty P3 is associated with the orienting response and its behavioural consequence of directing attention toward the task-irrelevant stimuli thereby resulting in impairment to ongoing task performance. The interpretation offered by Cortinas et al. (2008) is that the schizophrenic patients applied fewer attentional resources to the ongoing task and therefore the allocation of albeit reduced attentional resources to the deviant stimuli drastically affected behavioural performance. The foregoing suggests, therefore, that engagement in the task in addition to potential differences in auditory processing, might modulate the increased susceptibility to deviance distraction within high schizotypes. 
An alternative suggestion to the foregoing is that if the neural model is impoverished for high schizotypes, then any incoming sound is likely to fail to match the content of the neural model (because it is impoverished, even with repetition of the same sound) and can therefore trigger an OR. If it is not the case that the neural model is impoverished for high schizotypes, then then it is difficult to account for the need of more repetitions of the "deviant" to habituate to it as has been shown for high schizotypes (Vernon et al., 2005). The neural model may therefore exist for high schizotypes but with a content that is generally of low quality. As a result greater repetition of the same sound is required for that sound to be finally integrated within the model (e.g., Vernon et al., 2005). As such, when an incoming sound is compared with the content of the model, it fails to find its match, and in turn triggers an OR. Therefore, difficulties to habituate to a repeated sound do not necessarily mean that the OR to a new sound will be smaller. In fact, this habituation problem reflects difficulties in updating the neural model. A novel sound is likely to have no representation at all, and thus should trigger a large OR. As Vernon et al. (2005, p. 21) suggest:

“...if there are difficulties in the process of generating the initial neural template, after only a short interval, the template may be insufficiently formed to correctly match the incoming repeated stimulus. This could result in a mismatch between the ill-formed template and the repeated stimulus, leading the individual to orient to the repeated stimulus, which in turn would be identified as poor habituation."

In line with this idea, lower WMC has also been linked to impoverished habituation processes and a failure to inhibit the OR (Sörqvist et al., 2012). In this way, the symptoms of high values on unusual experiences and low WMC are rather similar, corroborated by the correlation between unusual experiences and WMC which demonstrated a negative relationship (cf. Table 2). Taken together, higher distractibility in high schizotypy might be related to two separate mechanisms, one of impoverished inhibition of the OR-as indexed 
by WMC and the unusual experiences subscale — and one of difficulties in withholding a sustained locus-of-attention - as indexed by the impulsivity subscale.

\section{Vulnerability of maintenance in working memory to distraction}

While we have this far considered that differences in auditory processing could underpin the differences in susceptibility to distraction via deviations, we must not neglect the potential role of differences in memory maintenance in modulating distractibility. A core part of the working memory system is to maintain mental representations for further processing in the face of interference from external (e.g., auditory, visual, and haptic distractors) and internal events (e.g., verbal and nonverbal thoughts including hallucination). Independent of modality (verbal or visual), and schizophrenic individuals show deficits that may be attributable an increased vulnerability of mental representations to distraction during their maintenance (Lee \& Park, 2005; the present study). A key process in visual-verbal and visual-spatial short-term remembering is rehearsal. For visual-verbal short-term memory, the vocal-motor system is co-opted to temporarily maintain a novel sequence of items to aid their eventual reproduction (Hughes \& Jones, 2005). Similarly, for visual-spatial short-term memory the oculomotor system is utilised: sequences of spatial location are maintained through corresponding eye-movements during delay periods (Tremblay, Saint-Aubin, \& Jalbert, 2006). One possibility, therefore, is that high schizotypes have more inchoate, less fluent, rehearsal skills regardless of domain. In this way it could take a high schizotype longer to consolidate or automatize a rehearsal cohort such that it becomes more resistant to distraction via an auditory deviant.

Elliott et al. (2016) demonstrated that rehearsal automatization and susceptibility to distraction are related. They varied the timing of the presentation of irrelevant sound in relation to the presentation of the to-be-remembered sequence. Irrelevant sound presented during the last $5 \mathrm{~s}$ of a 10 -s presentation or throughout the first $5 \mathrm{~s}$ of a 10 -s retention interval 
caused significantly more disruption than sound presented five seconds before the presentation of the list, the first five seconds of presentation and the final five seconds of retention. Elliott et al. (2016) proposed that rehearsal cohorts become increasingly stable across retention intervals and are thereby less susceptible to disruption by the presence of irrelevant sound. Moreover, Hughes, Hurlstone, and Jones (2016) report that deviation effects are more pronounced when the deviants are presented together with the earlier parts of the tobe-remembered sequence.

If the view that high schizotypes engage in nonfluent rehearsal is accurate, then it is possible that they will not reach a point at which their rehearsal is as fully automatized as a low schizotype would. If this is the case, then it would be expected that high schizotypes will be susceptible to disruption by irrelevant sound (including a deviant) even after a relatively extended period of rehearsing the same sequence, because the mental representation (or rehearsal cohort) is not yet consolidated and/or automatized (for a similar suggestion, see Lee \& Park, 2005). This could be tested by moving the deviant's position within the to-beremembered sequence (e.g., during a retention interval; Röer, Bell, \& Buchner, 2014). If rehearsal automatization is one factor in determining high schizotypes' increased vulnerability to distraction, then it may be possible to establish a different temporal gradient of vulnerability to disruption for high as compared with low schizotypes.

\section{Conclusion}

In summary, the results support the view that schizotypy, like schizophrenia, is associated with increased distractibility. One key finding is that the increased distractibility seen in people with positive schizotypy is not simply the result of co-variation between individual differences in schizotypy and individual differences in WMC. The other key finding is that functionally distinct mechanisms appear to underpin susceptibility to distraction and these are associated with WMC and the subscales of schizotypy. Further work 
is required to address whether the attentional deficit-as manifest in domain-general attentional capture-associated with higher schizotypy is a reliable cognitive marker of the vulnerability to the development of a schizophrenia-related disorder. 


\section{References}

Alain, C., Hargrave, R., \& Woods, D. (1998). Processing of auditory stimuli during visual attention in patients with schizophrenia. Biological Psychiatry, 44, 1151-1159.

Andreasen, N. C. (1999). A unitary model of schizophrenia: Bleuler's "fragmented phrene" as schizencephaly. Archives of General Psychiatry, 56, 781-787.

Bell, M. D., Byson, G., \& Wexler, B. (2001). Verbal working memory impairment in schizophrenia. American Journal of Psychiatry, 158, 660-661.

Braff, D. L. (1993). Information processing and attention dysfunctions in schizophrenia. Schizophrenia Bulletin, 19, 233-259.

Braff, D. L., Geyer, M. A., \& Swerdlow, N. R. (2001). Human studies of prepulse inhibition of startle: normal subjects, patient groups, and pharmacological studies. Psychopharmacology, 156, 234-258.

Cadenhead, K. S., Geyer, M. A., \& Braff, D. L. (1993). Impaired startle prepulse inhibition and habituation in patients with schizotypal personality disorder. American Journal of Psychiatry, 1, 1862-1867.

Carter, C. S., Robertson, L. C., Nordahl, T., Chadderjian, M., Kraft, L., O’Shora-Celaya, L. (1996). Spatial working memory deficits and their relationship to negative symptoms in unmedicated schizophrenia patients. Biological Psychiatry, 40, 930-932.

Cellard, C., Tremblay, S., Lehoux, C., \& Roy, M-A. (2007). Processing spatial-temporal information in recent-onset schizophrenia: The study of short-term memory and its susceptibility to distraction. Brain \& Cognition, 64, 201-207.

Claridge, G. (1997). Schizotypy: Implications for illness and health. Oxford University Press, London. 
Claridge, G., McGreedy, C., Mason, O., Bentall, R., Boyle, G., Slade, P., \& Popplewell, D. (1996). The factor structure of 'schizotypal' traits: A large replication study. British Journal of Clinical Psychology, 35, 103-115.

Cortinas, M., Corral, M-J., Garrido, G., Garolera, M., Pajares, M., \& Escera, C. (2008). Reduced novelty-P3 associated with increased behavioural distractibility in schizophrenia. Biological Psychology, 78, 253-260.

Cowan, N. (1995). Attention and memory: An integrated framework. New York: Oxford University Press.

Croft, R. J., Lee, A., Bertolot, J., Gruzelier, J. H. (2001). Associations of P50 suppression and desensitization with perceptual and cognitive features of "unreality" in schizotypy. Biological Psychiatry, 50, 441-446.

Dickman, S. J. (1993). Impulsivity and information processing. In W. McCown, M. Shure, \& J. Johnson, The impulsive client: theory research and treatment. Washington DC: American Psychological Association.

Dickman, S. J. (1996). Adverse (and beneficial) consequences of impulsive behavior: In S. R. Feldman, (Ed.) The Psychology of adversity. Amherst, MA: University of Massachusetts Press.

Dickman, S. J. (2000). Impulsivity, arousal and attention. Personality \& Individual Differences, 28, 563-581.

Elliott, E. M., Hughes, R. W., Brigianti, A. M., Joseph, T., Marsh, J. E., \& Macken, W. J. (2016). Distraction in verbal short-term memory: Insights from developmental differences. Journal of Memory \& Language, 88, 39-50.

Engle, R. W. (2002). Working memory capacity as executive attention. Current Directions in Psychological Science, 11, 19-23. 
Evans, L. H., Gray, N. S., \& Snowden, R. J. (2007). Reduced P50 suppression is associated with the cognitive disorganisation dimension of schizotypy. Schizophrenia Research, 97, 152-162.

Fuentes, L. J. (2001). Selective attention deficit in schizophrenia. Reviews in Neurology, 32, 387-391.

Fuentes, L. J., Boucart, M., Vivas, A. B., Alvarez, R., \& Zimmerman, M. A. (2000). Inhibitory tagging in inhibition of return is affected in schizophrenia: Evidence from the Stroop task. Neuropsychology, 14, 134-140.

Farmer, C. M., O'Donnell, B. F., Niznikiewicz, M. A., Voglmaier, M. M., McCarley, R. W., \& Shenton, M. E. (2000). Visual perception and working memory in schizotypal personality disorder. American Journal of Psychiatry, 157, 781-788.

Gold, J. M., Fuller, R. L., Robinson, B. M., Braun, E. L., \& Luck, S. J. (2007). Impaired topdown control of visual search in schizophrenia. Schizophrenia Research, 94, 148-155.

Gray, J. A., Feldon, J., Rawlins, J. N. P., Hemsley, D. R., \& Smith, A. D. (1991). The neuropsychology of schizophrenia. Behavioural Brain Research, 14, 1-20.

Hemsley, D. R. (1987). An experimental psychological model for schizophrenia. In: Hafner, H., Gattaz, W. F., Janzarik, W. (Eds.), Search for the causes of schizophrenia. Springer-Verlag, Berlin, pp. 179-188.

Hughes, R. W., Hurlstone, M. J., Marsh, J. E., Vachon, F., \& Jones, D. M. (2013). Cognitive control of auditory distraction: Impact of task difficulty, foreknowledge, and working memory capacity supports duplex-mechanism account. Journal of Experimental Psychology: Human Perception \& Performance, 39, 539-553.

Hughes, R. W., Hurlstone, M. J., \& Jones, D. M. (2016, under review). Increased load double-dissociates two forms of distraction in working memory: Differential engagements not limited working memory capacity? 
Hughes, R. W., \& Jones, D. M. (2005). The impact of order incongruence between a taskirrelevant auditory sequence and a task-relevant visual sequence. Journal of Experimental Psychology: Human Perception and Performance, 31, 316-327.

Hughes, R. W., Vachon, F., \& Jones, D. M. (2005). Auditory attentional capture during serial recall: Violations at encoding of an algorithm-based neural model? Journal of Experimental Psychology: Learning, Memory, \& Cognition, 31, 736-749.

Hughes, R. W., Vachon, F., \& Jones, D. M. (2007). Disruption of short-term memory by changing and deviant sounds: Support for a duplex-mechanism account of auditory distraction. Journal of Experimental Psychology: Learning, Memory, \& Cognition, $33,1050-1061$.

Kaplan, O., \& Lubow, R. E. (2011). Ignoring irrelevant stimuli in latent inhibition and Stroop paradigms: The effects of schizotypy and gender. Psychiatry Research, 186, 40-45.

Kane, M. J., Bleckley, M. K., Conway, A. R. A., \& Engle, R. W. (2001). A controlledattention view of WM capacity. Journal of Experimental Psychology: General, 130, 169-183.

Kane, M. J., \& Engle, R. W. (2003). Working-memory capacity and the control of attention: The contributions of goal neglect, response competition, and task set to Stroop interference. Journal of Experimental Psychology: General, 132, 47-70.

Lange, E. B. (2005). Disruption of attention by irrelevant stimuli in serial recall. Journal of Memory \& Language, 53, 513-531.

Lee, J., \& Park, S. (2005). Working memory impairments in schizophrenia: A meta-analysis. Journal of Abnormal Psychology, 114, 599-611.

Lipp, O. V., \& Vaitl, D. (1992). Latent inhibition in human Pavlovian differential conditioning: Effect of additional stimulation after preexposure and relation to schizotypal traits. Personality \& Individual Differences, 13, 1003-1012. 
Lubow, R. E., Ingberg-Sachs, Y., Zahlstein-orda, N. and Gewirtz, J. (1992). Latent inhibition in low and high " psy-chotic prone”" normal subjects. Personality \& Individual Differences, 13, 563-572.

Mason, O., Claridge, G., \& Jackson, M. (1995). New scales for the assessment of schizotypy. Personality \& Individual Differences, 18, 7 - 13.

MacDonald, N. (1960). The other side: Living with schizophrenia. Canadian Medical Association Journal, 82, 218-221.

Mednick, S. A., \& McNeil, T. (1968). Current methodology in research on the etiology of schizophrenia. Psychological Bulletin, 70, 681-693.

McGhie, A., \& Chapman, J. (1961). Disorders of attention and perception in early schizophrenia. British Journal of Medical Psychology, 34, 103-116.

McGurk, S. R., Carter, C., Goldman, R., Green, M. F., Marder, S. R., Xie, H., et al. (2005). The effects of clozapine and risperidone on spatial working memory in schizophrenia. American Journal of Psychiatry, 162, 1013-1016.

Mirsky, A. F., \& Duncan, C. C. (1986). Etiology and expression of schizophrenia: neurobiological and psychosocial factors. Annual Review of Psychology, 37, 291-319.

Miyake, A., Friedman, N. P., Rettinger, D. A., Shah, P., \& Hegarty, M. (2001). How are visuospatial working memory, executive functioning, and spatial abilities related? A latent-variable analysis. Journal of Experimental Psychology: General, 130, 621-640.

Näätänen, R., Tervaniemi, M., Sussman, E., Paavilainen, P., \& Winkler, I. (2001). Primitive intelligence in the auditory cortex. Trends in Neuroscience, 24, 283-287.

Nestor, P. G., \& O’Donell, B. F. (1998). The mind adrift: attentional disregulations in schizophrenia. In R. Parasuraman (Ed.), The attentive brain. Cambridge, MA: MIT Press. 
O’Driscoll, G. A., Strakowski, S. M., Alpert, N. M., Mathysse, S. W., Rauch, S. L., Levy D. L., et al. (1998): Differences in cerebral activation during smooth pursuit and saccadic eye movements using positron-emission tomography. Biological Psychiatry, 44, 685689.

O’Flynn, K., Gruzelier, J. H., Bergman, A., \& Siever, L. J. (2003). The schizophrenia spectrum personality disorders. In. S. R. Hirsch \& D. Weinberger (Eds,), Schizophrenia (pp. 80-100). Chichester, England: Blackwell.

Öhman, A., Nordby, H., \& d'Elia, G. (1986). Orienting and schizophrenia: stimulus significance, attention, and distraction in a signalled reaction time task. Journal of Abnormal Psychology, 95, 326-334.

Oltmans, T. F. (1978). Selective attention in schizophrenic and manic psychoses: The effect of distraction on information processing. Journal of Abnormal Psychology, 87, 212225.

Oltmans, T. F., \& Neale, J. M. (1975). Schizophrenic performance when distractors are present: Attentional deficit or differential task difficulty? Journal of Abnormal Psychology, 84, 205-209.

Park, S., \& Gooding, D. C. (2014). Working memory impairment as an endophenotypic marker of schizophrenia diathesis. Schizophrenia Research: Cognition, 1, 127-136.

Pelletier, M.-F., Hodgetts, H. M., Lafleur, M. F., Vincent, A., \& Tremblay, S. (2013). Vulnerability to the irrelevant sound effect in adult ADHD. Journal of Attention Disorders.

Pirkola, T., Tuulio-Henriksson, A., Glahn, D., Kieseppa, T., Haukka, J., Kaprio, J., et al. (2005). Spatial working memory in twins with schizophrenia and bipolar disorder. Biological Psychiatry, 58, 930-936. 
Poreh, A.M., Ross, T. P., Whitman, R. D. (1995). Reexamination of executive functions in psychosis-prone college students. Personality and Individual Differences 18, 535-539.

Raine, A., Benishay, D., Lencz, T., \& Scarpa, A. (2007). Abnormal orienting in schizotypal personality disorder. Schizophrenia Bulletin, 23, 75-82.

Rawlings, D., \& Goldberg, M. (2001). Correlating a measure of sustained attention with a multidimensional measure of schizotypal traits. Personality \& Individual Differences, $31,421-431$.

Röer, J. P., Bell, R., \& Buchner, A. (2014). What determines auditory distraction? On the roles of local auditory changes and expectation violations. PLoS One, 9, e84166.

Schröger, E. (1997). On the detection of auditory deviants: A preattentive activation model. Psychophysiology, 34, 245-257. doi:10.1111/j.1469-8986.1997.tb02395.x

Siddle, D. A. (1991). Orienting, habituation, and resource allocation: an associative analysis. Psychophysiology, 28, 245-259.

Siever, L. J., Koeningsberg, H. W., Harvey, P., Mitropoulou, V., Laruelle, M., Abi-Dargham, A., Goodman, M., Bauchsbaum, M. (2002). Cognitive and brain function in schizotypal personality disorder. Schizophrenia Research, 54, 157-167.

Smith, E. E., Jonides, J., \& Koeppe, R. A. (1996). Dissociating verbal- and spatial-working memory using PET. Cerebral Cortex, 6, 11-20.

Smith, C. W., Park, S., \& Cornblatt, B. (2006). Spatial working memory deficits in adolescents at clinical high risk for schizophrenia. Schizophrenia Research, 81, 211215.

Sokolov, E. N. (1963). Perception and the conditioned reflex. New York: Macmillan.

Sörqvist, P. (2010). High working memory capacity attenuates the deviation effect but not the changing-state effect: Further support for the duplex-mechanism account of auditory distraction. Memory \& Cognition, 38, 651-658. 
Sörqvist, P., \& Marsh, J. E. (2015). How concentration shields against distraction. Current Directions in Psychological Science, 24, 267-272.

Sörqvist, P., Marsh, J. E., \& Nöstl, A. (2013). High working memory capacity does not always attenuate distraction: Bayesian evidence in support of the null hypothesis. Psychonomic Bulletin \& Review, 20, 897-904.

Sörqvist, P., Nöstl, A., \& Halin, N. (2012). Working memory capacity modulates habituation rate: Evidence from a cross-modal auditory distraction paradigm. Psychonomic Bulletin \& Review, 19, 245-250.

Sörqvist, P., \& Rönnberg, J. (2014). Individual differences in distractibility: An update and a model. PsyCh Journal, 3, 42-57.

Sörqvist, P., Stenfelt, S., \& Rönnberg, J. (2012). Working memory capacity and visual-verbal cognitive load modulates auditory-sensory gating in the brainstem: toward a unified view of attention. Journal of Cognitive Neuroscience, 24, 2147-2154.

Tremblay, S., Parmentier, F. B. R., Guérard, K., Nicholls, A., \& Jones, D. M. (2006). A spatial modality effect in serial memory. Journal of Experimental Psychology: Learning, Memory, \& Cognition, 32, 1208-1215.

Unsworth, N., Heitz, R. P., Schrock, J. C., \& Engle, R. W. (2005). An automated version of the operation span task. Behavior Research Methods, 37, 498-505.

Vachon, F., Hughes, R. W., \& Jones, D. M. (2012). Broken expectations: Violation of expectancies, not novelty, captures auditory attention. Journal of Experimental Psychology: Learning, Memory, \& Cognition, 38, 164-177.

Vachon, F., Labonté, K., \& Marsh, J. E. (2014). Attentional capture by deviant sounds: A general form of distraction? Manuscript in preparation. 
Van den Bosch, R.J. (1995). Context and cognition in schizophrenia. In J. A. Den Boer, \& H. G. M. Westenberg (Eds.), Advances in the neurobiology of schizophrenia. New York: John Wiley \& Sons.

Vernon, D., Haenschel, C., Dwivedi, P., \& Gruselier, J. (2005). Slow habituation of induced gamma and beta oscillations in association with unreality experiences in schizotypy. International Journal of Psychophysiology, 56, 15-24.Vollerma, M. G., \& van der Bosch, R. J. (1995). The multidimensionality of schizotypy. Schizophrenia Bulletin, $21,19-31$.

Voronin, L. G., \& Sokolov, Y. N. (1960). Cortical mechanisms of the orienting reflex and its relation to the conditioned reflex. Electroencephalography \& Clinical Neurophysiology, 13, 335-346. 


\section{Table 1}

The overall mean and standard errors for Working Memory Capacity (WMC), overall schizotypy and the four schizotypy subscales of the O-LIFE questionnaire (Mason et al., 1995). For these individual differences measures, comparisons are made (via student t-tests) between those scoring high, and those scoring low, on the overall schizotypy measure based on a median split ( $N=42$ in both groups).

\section{Overall High schizotypy Low schizotypy}

\begin{tabular}{lccccccc} 
Variable & $M$ & $S E$ & $M$ & $S E$ & $M$ & $S E$ & Student $t$ \\
\hline Operation span & 44.48 & 1.67 & 40.9 & 1.88 & 48.05 & 2.67 & $2.19^{*}$ \\
Overall schizotypy & 34.24 & 1.5 & 45.33 & 1.3 & 23.14 & 1.19 & $12.60^{*}$ \\
$\quad$ & & & & & & & \\
$\quad$ Schizotypy subscales & & & & & & & \\
Unusual Experiences & 8.75 & 0.63 & 12.21 & 0.81 & 5.23 & 0.60 & $6.90^{*}$ \\
Impulsive Nonconformity & 8.2 & 0.37 & 9.92 & 0.53 & 6.48 & 0.37 & $5.36^{*}$ \\
Introvertive Anhedonia & 5.88 & 0.49 & 7.81 & 0.75 & 3.95 & 0.49 & $4.29 *$ \\
Cognitive Disorganization & 11.43 & 0.59 & 15.43 & 0.51 & 7.43 & 0.60 & $10.19 *$ \\
\hline
\end{tabular}

Note: $t$ statistic is the test of the difference between high and low schizotypy groups

$* p<.05$ 
Table 2

Intercorrelations amongst the six individual differences variables.

\begin{tabular}{lccccc}
\hline \multicolumn{1}{c}{ Variable } & 1 & 2 & 3 & 4 & 5 \\
\hline 1. Operation span & - & & & & \\
2. Overall schizotypy & $-.22^{*}$ & - & & & \\
3. Unusual Experiences & $-.28^{*}$ & $.79 *$ & - & & \\
4. Impulsive Nonconformity & -.12 & $.61 *$ & $.32 *$ & - & - \\
5. Introvertive Anhedonia & -.09 & $.53^{*}$ & $.19 \dagger$ & .03 & $.30 *$ \\
6. Cognitive Disorganization & -.12 & $.88^{*}$ & $.59 *$ & $.58 *$ & \\
\hline
\end{tabular}

$* p<.05, \dagger p<.05$ (one tailed) 


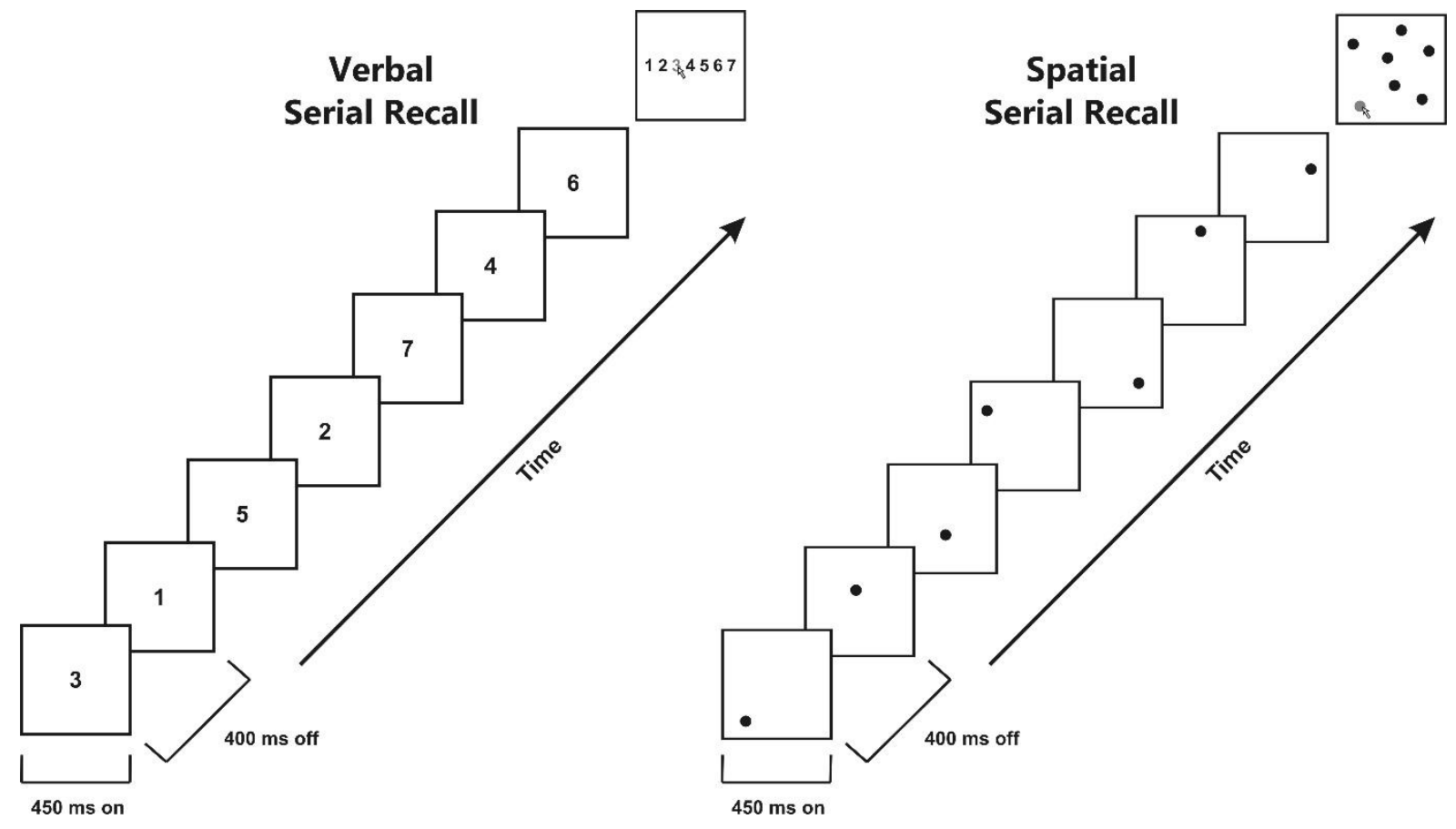

Figure 1. Schematic diagram of the to-be-remembered lists used in the verbal and spatial tasks of the experiment: Representation of the sequential presentation of the seven items (digits or dots) and of the response procedure. The illustration is not to scale. 

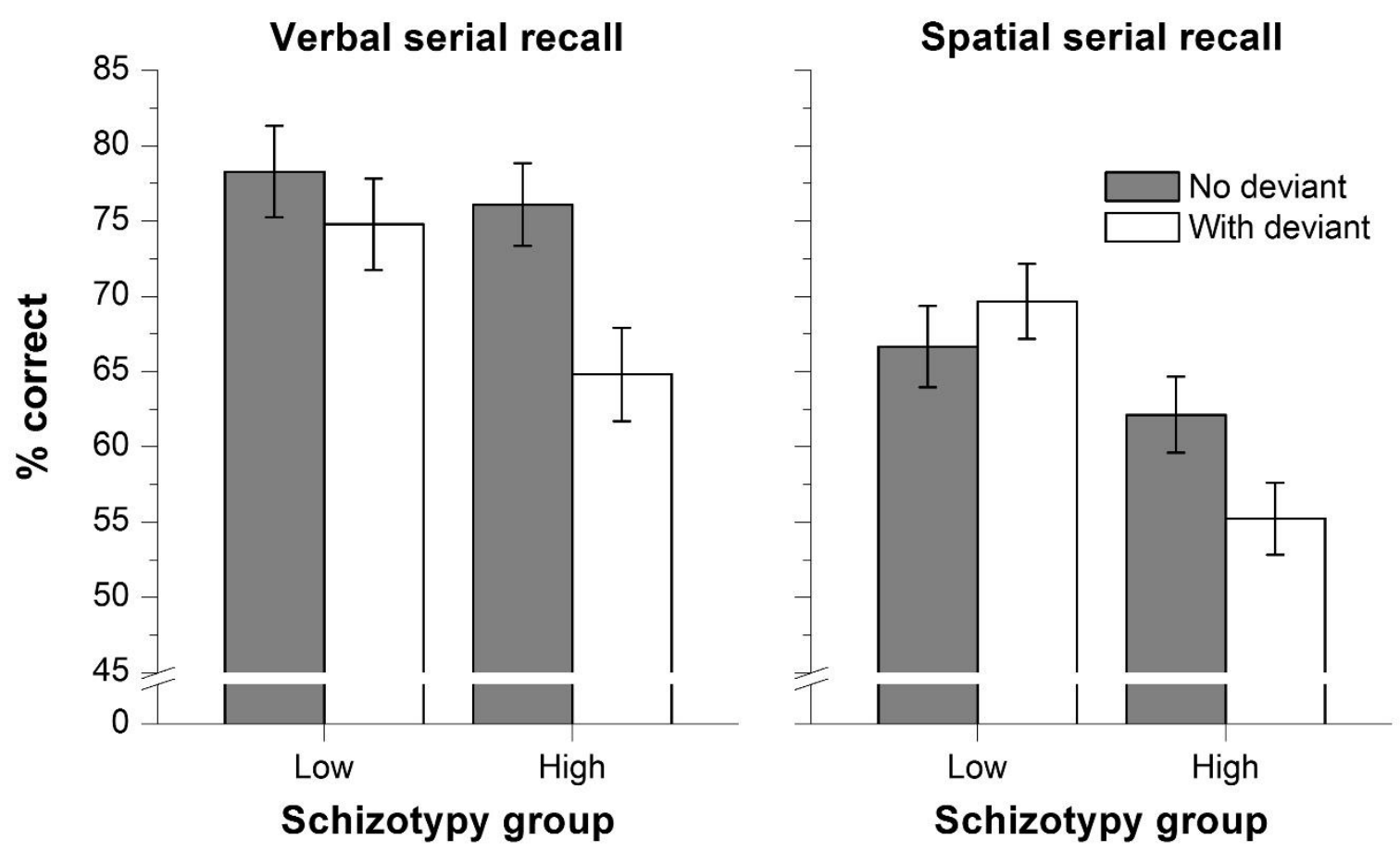

Figure 2. Percentages of correct responses for 'no deviant' and 'with deviant' trials in each schizotypy group under verbal (left panel) and spatial serial recall (right panel). Error bars represent the standard error of the mean. 

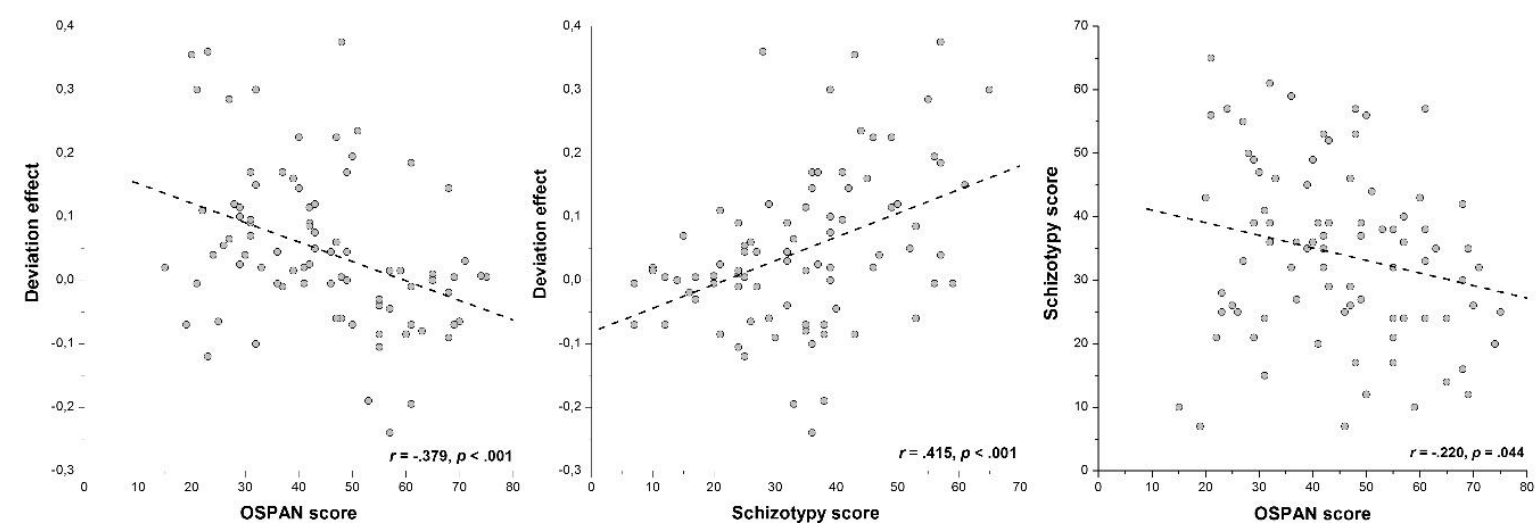

Figure 3. Scatterplots showing the relationship between: the size of the deviation effect averaged across the two serial recall tasks and working memory capacity (as measured by OSPAN; left panel) and the schizotypy score (middle panel). The right panel shows the relationship between schizotypy score and working memory capacity $(N=84)$. The dashed line represents the best linear fit between the two variables. 

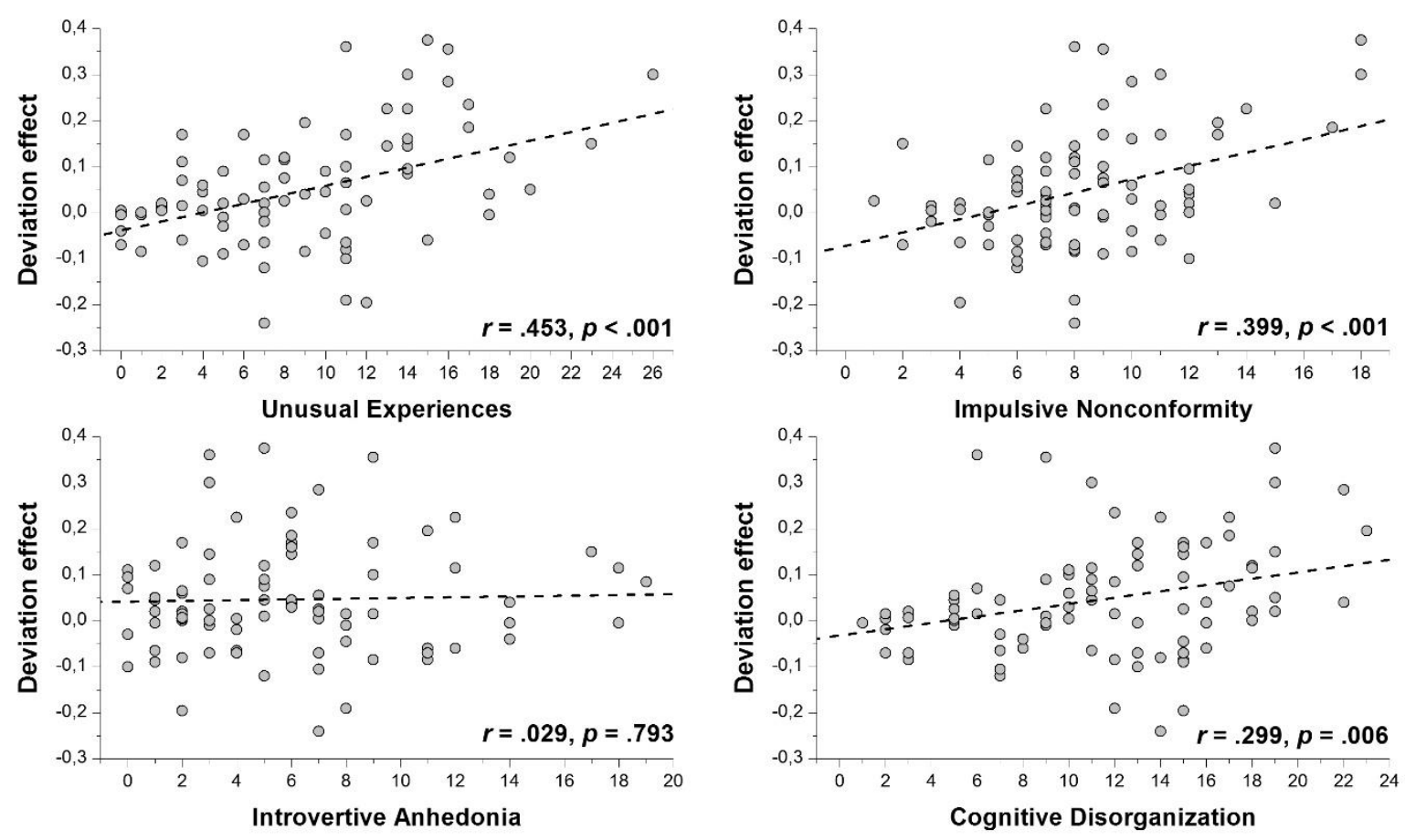

Figure 4. Scatterplots showing the relationship between the size of the deviation effect averaged across the two serial recall tasks and the score obtained in each of the four schizotypy subscales $(N=84)$. The dashed line represents the best linear fit between the two variables. 